POS PROCEEDINGS

\title{
Mass measurements of stored exotic nuclei
}

\author{
Yuri A. Litvinov* \\ Max-Planck Institut für Kernphysik, Saupfercheckweg 1, 69117 Heidelberg, Germany \\ GSI Helmholtzzentrum für Schwerionenforschung, Planckstrasse 1, 64291 Darmstadt, Germany \\ E-mail: y.litvinov@gsi.de
}

\section{Klaus Blaum}

Max-Planck Institut für Kernphysik, Saupfercheckweg 1, 69117 Heidelberg, Germany

Ruprecht-Karls-Universität Heidelberg, 69120 Heidelberg, Germany

E-mail: klaus.blaum@mpi-hd.mpg.de

\section{Xinwen Ma}

Institute of Modern Physics, Chinese Academy of Sciences, Lanzhou, 730000, China

E-mail: x.ma@impcas.ac.cn

\section{Philip M. Walker}

Department of Physics, University of Surrey, Guildford, Surrey GU2 7XH, UK

E-mail: p.walkeresurrey.ac.uk

\section{Nicolas Winckler}

Max-Planck Institut für Kernphysik, Saupfercheckweg 1, 69117 Heidelberg, Germany

E-mail: n.winckler@gsi.de

\begin{abstract}
Knowledge of atomic masses is indispensable in nuclear structure and nuclear astrophysics. In the last two decades, majority of experimental data on nuclear masses stems from measurements of stored ions. The ions can be stored at low energies in a Penning trap or at significantly higher energies in a storage ring. In both cases revolution frequencies of ions trapped in a confining magnetic field are determined which enables high-precision mass measurements. In this contribution we sketch the experimental techniques and recent results. Future perspectives at the new generation radioactive beam facilities are outlined.
\end{abstract}

11th Symposium on Nuclei in the Cosmos, NIC XI

July 19-23, 2010

Heidelberg, Germany

\footnotetext{
*Speaker.
} 


\section{Introduction}

Importance of nuclear mass is evident since it reflects the total energy of the nucleus, the manybody object in which the strong, weak, and electromagnetic fundamental interactions take place by acting between the nucleons composing it. The nuclear mass surface is a very sensitive probe on which nuclear structure effects, such as shell structure, nucleon-nucleon correlations or changes in deformation are clearly visible [1]. Accurate nuclear masses can constrain nuclear models, see e.g. [2]. It has been shown that ground state masses reveal the structure of excited collective states [3]. Nucleon separation energies are derived from nuclear masses and are a key input for nucleosynthesis calculations [4]. Open questions such as, e.g., changes of nuclear shell structure far from the valley of $\beta$-stability involve nuclei with yet unknown masses. However, they are difficult to investigate due to their small production cross-sections and short lifetimes and require very efficient and fast experimental techniques. Two kinds of such experimental techniques routinely used for direct mass measurements are the Penning trap and storage ring mass spectrometry [5, 6].

\section{Penning trap mass spectrometry}

Penning trap facilities are planned or are in operation at the majority of radioactive beam facilities (see, e.g., [5]). A schematic illustration of a Penning trap is shown on the left panel of Figure 1. The trap is located inside a strong magnetic field of typically $B=6$ to $9 \mathrm{~T}$. In order to trap the ions, a voltage $U_{0}$ is applied to the ring and to the endcap electrodes, thus enabling a weak quadrupole electrostatic potential. Typically, singly charged ions are employed like, for instance, at ISOLTRAP in Geneva [9], JYFLTRAP in Jyväskylä [10], Canadian Penning trap at the Argonne National Laboratory [11], SHIPTRAP in Darmstadt [12], or LEBIT in East Lansing [13]. However, the precision of mass measurements can be considerably increased if highly-charged ions are used [14]. The TITAN setup in Vancouver [15] is a trap facility coupled to Electron-Beam Ion Trap (EBIT) which utilizes highly-charged ions. Another facility for measurements with highly-charged ions is the MLLTRAP which is being constructed in Garching [16]. The ion's motion in the trap is composed of three eigen-motions and it can be selectively excited by a quadrupole RF field
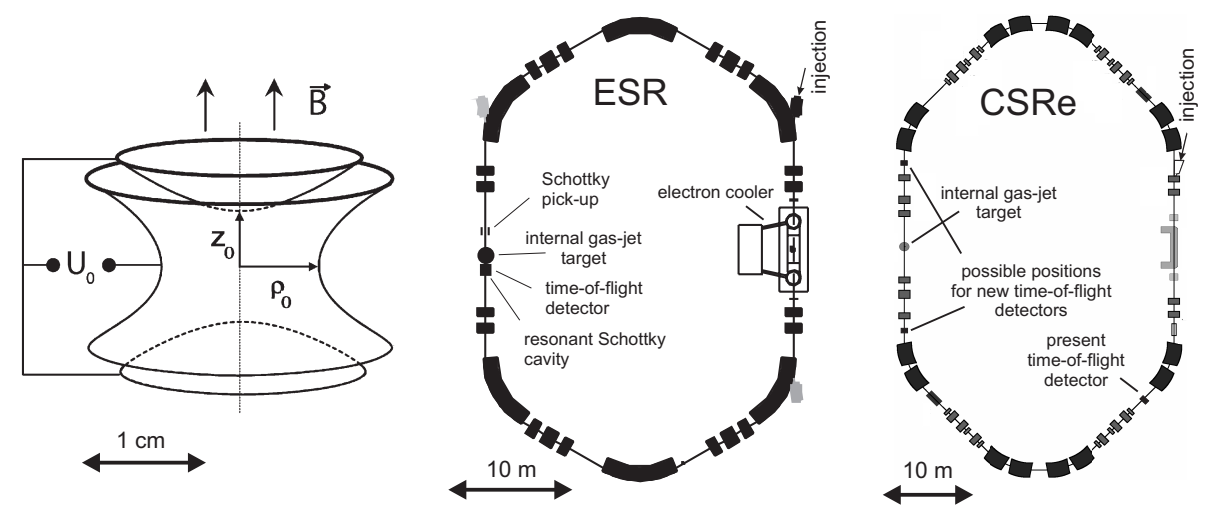

Figure 1: Schematic illustration of a Penning trap with hyperbolic electrodes (left) [5], the experimental storage ring ESR at GSI (middle) [7] and the cooler-storage ring CSRe at the IMP (right) [8]. 
applied to the segmented ring electrode. If the applied frequency matches the cyclotron frequency of the ions $\left(f_{c}=q B /(2 \pi m)\right)$, the radial kinetic energy is maximized. The energy is typically determined by extracting the ions from the trap and measuring their time of flight to the timing detector placed outside the magnetic field. The radial energy of the ions is transformed into the axial energy when the ions pass the magnetic field gradient [5]. The magnetic field inside the trap is calibrated by employing ions with well-known masses. Since recently, clusters of carbon atoms have been successfully used for calibration purposes [17]. TRIGA-TRAP is being commissioned at the nuclear research reactor TRIGA in Mainz [18]. Here, in addition to time-of-flight detection, the non-destructive Fourier-transform ion-cyclotron-resonance technique has been implemented. In this method, the cyclotron frequency of the oscillating ion is measured via the induced image currents in the segmented ring electrode [19].

\section{Storage ring mass spectrometry}

There are presently two facilities worldwide which employ high-energy storage rings for accurate mass measurements [20]. The radioactive beam facility at GSI in Darmstadt is a combination of the high-energy heavy-ion synchrotron SIS, the in-flight fragment separator FRS, and the coolerstorage ring ESR [6]. The ESR facility is schematically illustrated in the middle panel of Figure 1 [7]. The facility at IMP in Lanzhou is based on the same principle as the one at GSI. It consists of the synchrotron CSRm which is coupled to the storage ring CSRe via the in-flight fragment separator RIBLL2 [8]. The CSRe facility is illustrated in the right panel of Figure 1.

Radioactive ions of interest are produced at high energies (several hundred $\mathrm{MeV} / \mathrm{u}$ ), separated in-flight and stored in a storage ring [21,22]. Dependending on the specific experimental requirements, either cocktail or clean mono-isotopic beams can be prepared. The cocktail beams are ideally used for the mass measurements. Separation of mono-isotopic beams is often essential for accurate lifetime determination [23]-[28] or reaction studies with internal targets [29, 30].

In first-order approximation the revolution frequencies $f$ of the stored ions can be related to their mass-to-charge ratios $(\mathrm{m} / \mathrm{q})$ by the following expression:

$$
\frac{\Delta f}{f}=-\frac{1}{\gamma_{t}^{2}} \frac{\Delta(m / q)}{(m / q)}+\left(1-\frac{\gamma^{2}}{\gamma_{t}^{2}}\right) \frac{\Delta v}{v},
$$

where $\gamma$ is the relativistic Lorentz factor and $\gamma_{t}$ is the transition point of a storage ring [31]. The latter quantity is constant for a given ion-optical setting of the ring. According to the above equation a measurement of the frequencies of the stored ions allows the determination of their $m / q$ values only if the second term on the right-hand side can be neglected. However, secondary beams have a velocity spread $\Delta v / v$ of the order of a few percent which is an inevitable consequence of the nuclear reaction process [32]. Two complementary methods to minimize the term containing the velocity spread in Eq. (3.1) have been developed [6].

In Schottky mass spectrometry (SMS) the velocity spread is reduced by stochastic [33] and electron cooling [34], which force all stored ions towards the same mean velocity. The cooling process lasts a few seconds and the nuclides with half-lives longer than about $1 \mathrm{~s}$ can be investigated. Typical revolution frequencies are about $2 \mathrm{MHz}$ in the ESR and about $1.5 \mathrm{MHz}$ in the CSRe. Each ion induces at each revolution a mirror charge on a couple of electrostatic pick-up electrodes, 


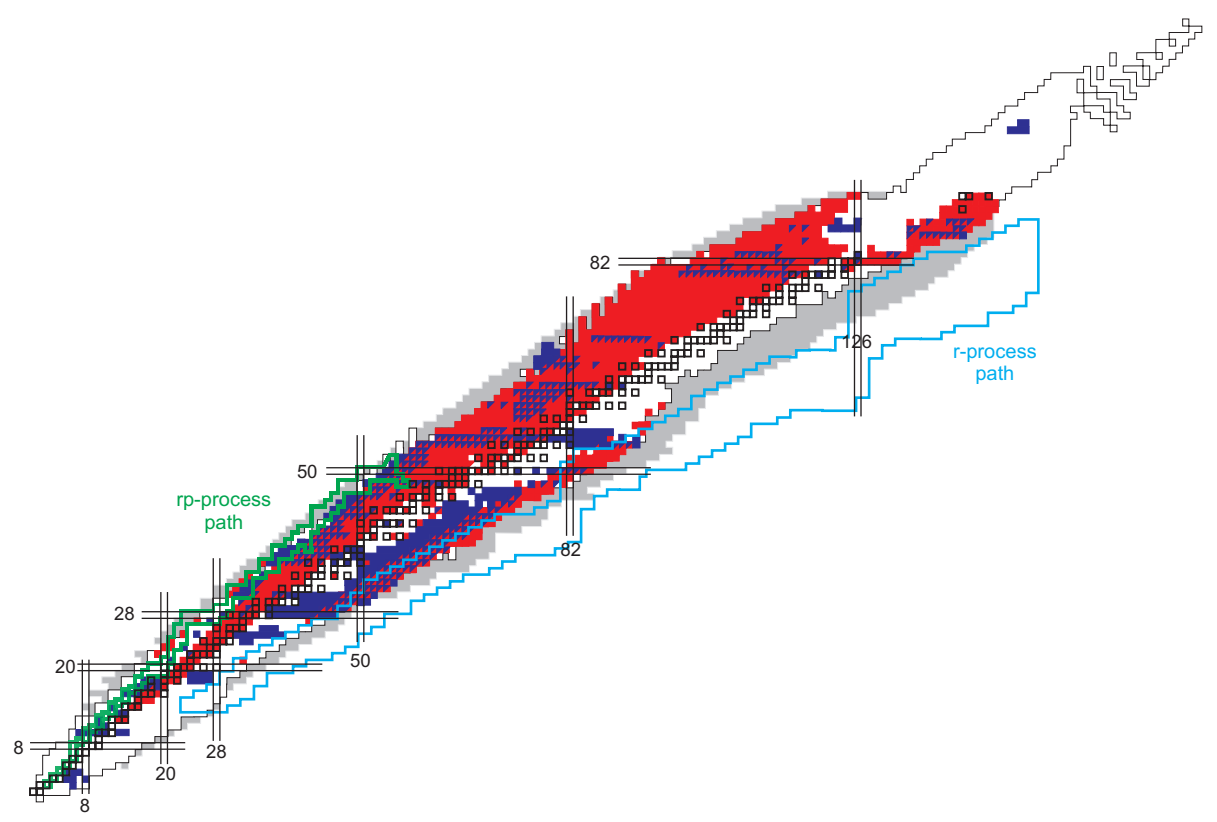

Figure 2: The chart of the nuclides. The squares outlined with black lines indicate stable nuclides. White area illustrates known isotopes. Nuclei shown in red and blue color represent masses measured with storage ring or Penning trap mass spectrometry, respectively. If the nucleus has been investigated with both methods, the square is divided into two correspondingly colored triangles. The data are taken from [41]-[69]. The paths of astrophysical rp- and r-processes of nucleosynthesis in stars are indicated. The area shaded in gray shows the nuclei whose mass measurements are planned at FAIR [70].

which yields, after Fast Fourier transform, a spectrum of revolution frequencies [35]. In 2010 a new Schottky detector based on a resonator cavity has been commissioned in the ESR [36].

In the Isochronous mass spectrometry (IMS), the ring is tuned into the isochronous ion-optical mode and the ions of interest are injected into the ESR or CSRe with $\gamma=\gamma_{t} \approx 1.41$ or 1.395 , respectively [20]. In such mode, a faster ion of a given ion species moves on a longer orbit and a slower ion of the same ion species moves on a shorter orbit, so that the velocity spread is compensated by the lengths of the closed orbits. The revolution frequencies are measured with a dedicated time-of-flight detector [37]. The fragments do not require cooling and the nuclei with half-lives as short as $10 \mu s$-about 20 revolutions in the ring-can be measured with this method. Present and planned positions of the time-of-flight detectors can be seen in Figure 1. The two detectors in the straight section of the CSRe are intended for in-ring measurement of particle velocities.

\section{Results}

Penning trap and storage ring mass spectrometry are highly complementary. On one side, the highest mass accuracies can be achieved with Penning traps [5]. On the other side, the storage rings are ideal instruments for broad-band mass measurements in which a few ten nuclides are studied simultaneously [38, 39, 40, 69]. The accurate mass values determined with Penning traps are of utmost importance for calibration purposes in the analysis of storage ring experiments. The chart 
of nuclides in Figure 2 illustrates the present status of mass measurements conducted with the two techniques [41]. The relative mass accuracy of the SMS amounts presently to $\Delta(m) / m \approx 1.5 \cdot 10^{-7}$. Somewhat better mass accuracy of about $\Delta(m) / m \approx 4 \cdot 10^{-8}$ can be reached in special cases by using narrow frequency ranges and well known masses for calibration. For example, the mass excess value $M E=-84376(4) \mathrm{keV}$ [71] has been obtained for the isomeric state ${ }^{93 m}$ Mo which is in excellent agreement with the literature value $M E=-84378(4) \mathrm{keV}$ [42]. The achieved mass resolving powers of IMS are about $R \approx 10^{5}$ (ESR) and $R \approx 5 \cdot 10^{4}$ (CSRe), which have to be compared to $R \approx 10^{6}$ obtained in SMS experiments. Restricting the acceptance of the injected beam in the IMS experiments allows increasing of the resolving power [72]. First IMS experiments have been performed at the CSRe [73, 74]. The masses of ${ }^{63} \mathrm{Ge},{ }^{65} \mathrm{As}$ and ${ }^{67} \mathrm{Se}$ and ${ }^{71} \mathrm{Kr}$ have been measured for the first time. In order to increase the resolving power, the in-ring velocity measurement of each stored ion is planned. For this purpose, two time-of-flight detectors will be installed in one of the straight sections (see Figure 1). In Penning trap experiments the relative mass accuracy of a few times $10^{-9}$ is routinely achieved [75, 76].

Both Penning trap and storage ring approaches are highly sensitive. Several new isotopes ${ }^{235} \mathrm{Ac}$ [77], ${ }^{236} \mathrm{Ac},{ }^{224} \mathrm{At},{ }^{221} \mathrm{Po},{ }^{222} \mathrm{Po}$ and ${ }^{213} \mathrm{Tl}$ [78] have been discovered in the ESR where their masses and lifetimes were determined. The mass values for the chain of radon isotopes have been determined at ISOLTRAP where the new isotope ${ }^{229} \mathrm{Rn}$ has been discovered [79]. Revolution frequency of a single stored ion can be measured with the SMS [80, 81]. For instance the mass value of ${ }^{208} \mathrm{Hg}$ (ME=-13265(31) keV) has been determined from a single stored hydrogen-like ion [82]. This new mass value has been used to estimate the interaction strength of the last two protons with the last two neutrons in ${ }^{210} \mathrm{~Pb}$. The obtained value $\delta V_{p n}\left({ }^{210} \mathrm{~Pb}\right)=165.2(10) \mathrm{keV}$ is about 2.5 times smaller than the $\delta V_{p n}\left({ }^{208} \mathrm{~Pb}\right)=426.8(5) \mathrm{keV}$. This sudden decrease just after the magic neutron number $N=126$ confirms the expectations based on the spatial overlap of the valence orbits in this region and their relation to very general properties of the shell structure near stability [82].

Detecting single ions is of a great advantage for resolving low-lying isomeric states since the ion can only be present in one or the other state [83]. Thus, ground or isomeric states can be
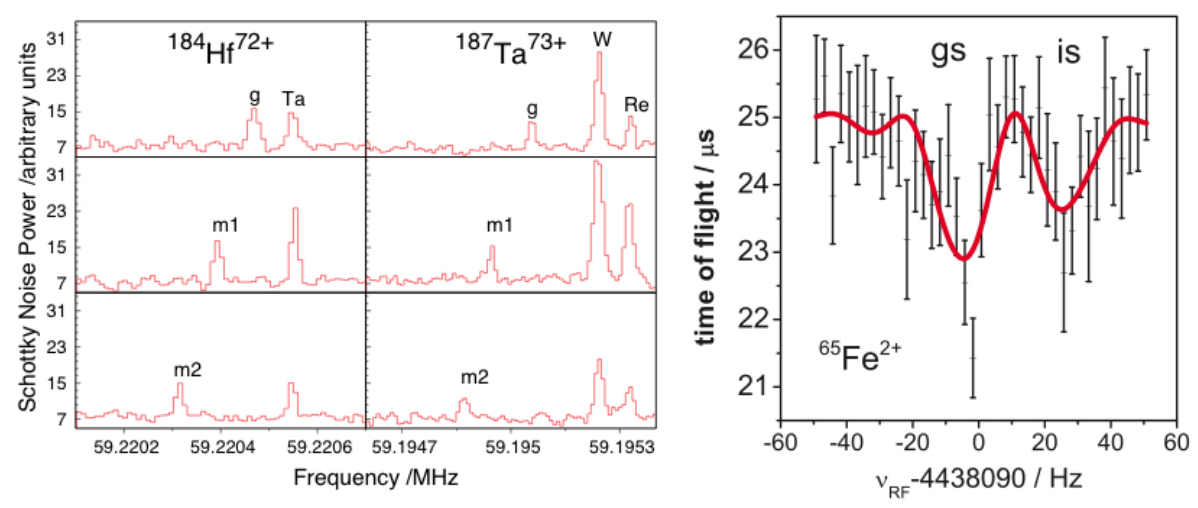

Figure 3: Discovery of nuclear isomeric states. Left: New isomeric states ${ }^{184 m 2} \mathrm{Hf},{ }^{187 m 1} \mathrm{Ta}$ and ${ }^{187 m 2} \mathrm{Ta}$ which have been observed in the ESR [85]. Right: mass measurement of ground and isomeric states in ${ }^{65} \mathrm{Fe}$ ions conducted at LEBIT [86] (courtesy of M. Block). 

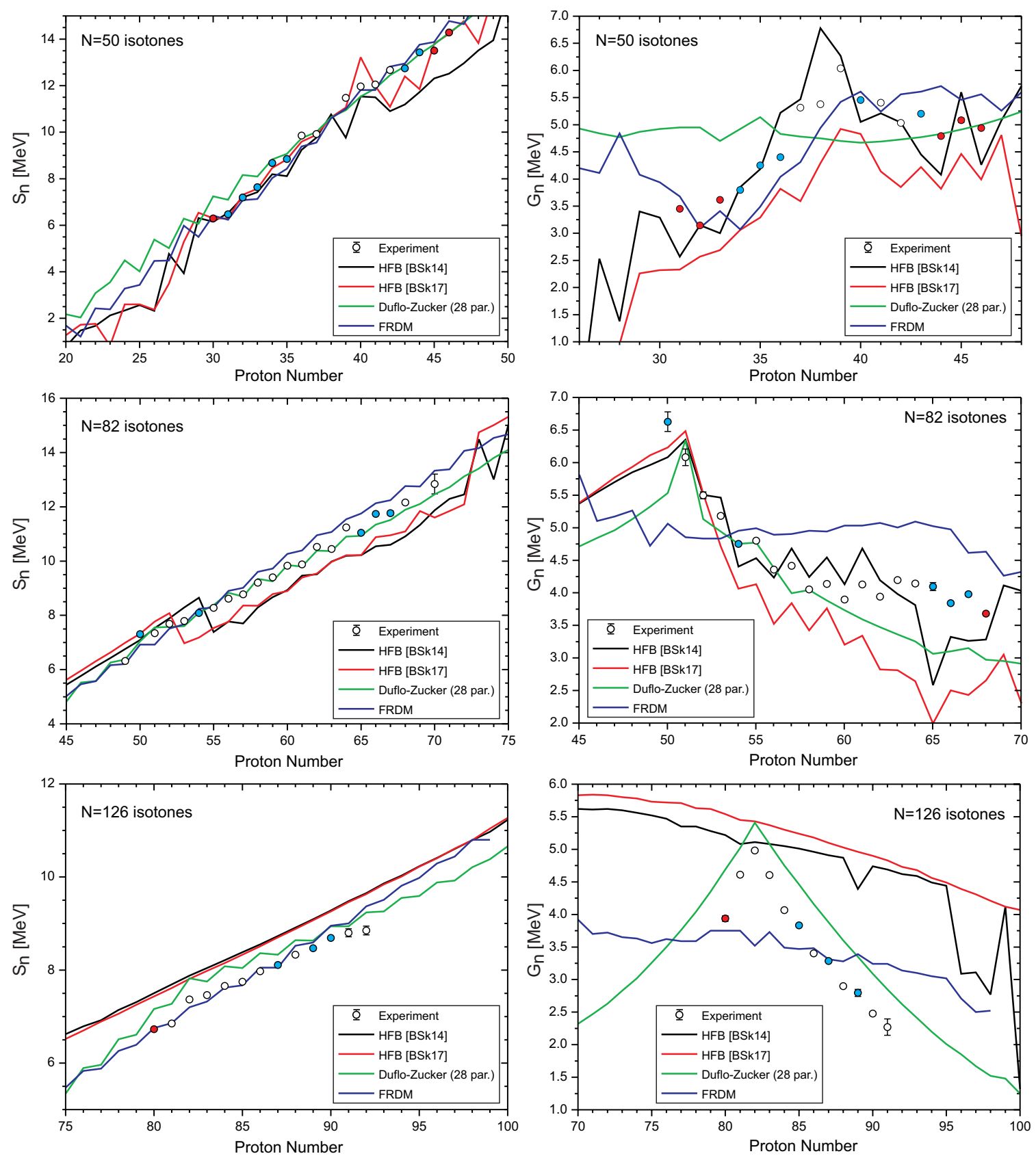

Figure 4: Left: experimental and calculated neutron separation energies for $N=50,82$ and 126 isotones as a function of proton number, shown on the upper, middle and lower panels, respectively. Right: experimental and calculated neutron shell gaps for $N=50,82$ and 126 isotones as a function of proton number (upper, middle and lower panels, respectively). The experimental data indicated with open symbols are from the latest Atomic Mass Evaluation (AME'03) [42]. New values and values with improved mass accuracy are indicated with red and blue symbols, respectively. The new values since the publication of the AME'03 are taken from Refs. [43]-[58]. The theoretical models are indicated in the legend and are described in detail in Refs. [89, 90, 91, 92]. The error bars, if not indicated, are within the symbol size. 


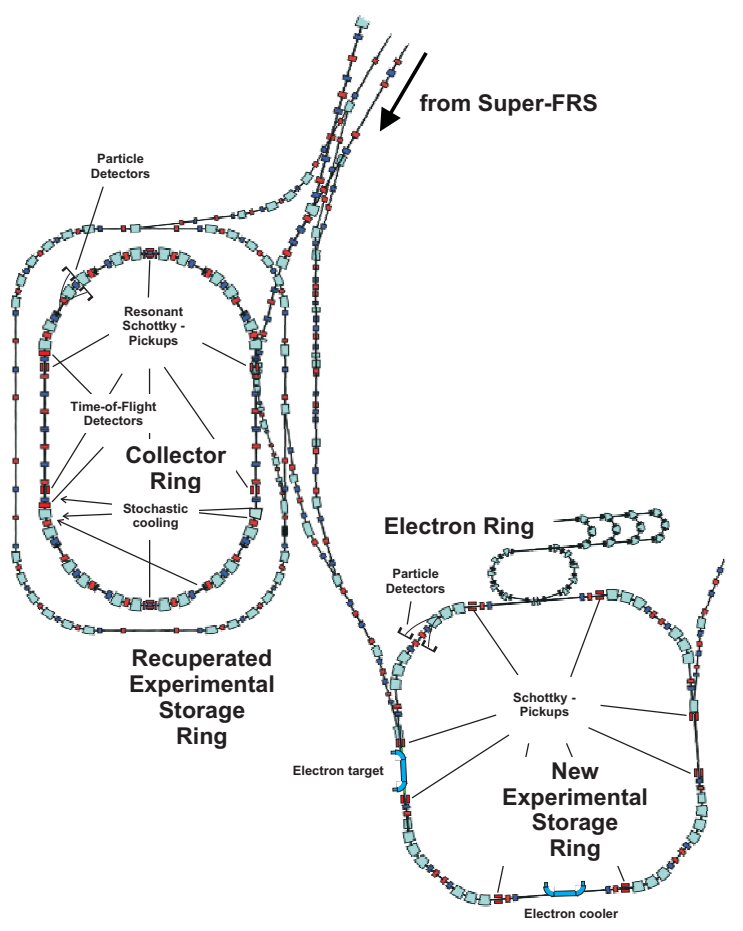

Figure 5: New storage ring complex planned at the new generation radioactive beam facility FAIR in Darmstadt. Exotic nuclei separated with the Super-FRS will be injected into the Collector Ring (CR) tuned into isochronous ion-optical mode, thus enabling IMS measurements [70, 97]. Two time-of-flight detectors will be installed to ensure in-ring velocity measurements of the stored particles [83]. Longer-lived nuclides will be stochastically pre-cooled and transferred via RESR into the New Experimental Storage Ring (NESR), where the masses of electron cooled ions will be measured with higher accuracy employing the SMS method. Multiple Schottky resonant cavities or pick-ups will be installed in both the CR and the NESR rings in order to enable fast SMS [70]. Preparation of pure isomeric beams for experiments with internal electron or gas targets is planned [70, 98].

assigned even for very small excitation energies which is not possible under the condition when both states are simultaneously populated. Single ${ }^{125} \mathrm{Ce}^{58+}$ ions have been observed in the ESR at two distinguished revolution frequencies. In this way, a long-lived isomeric state in ${ }^{125} \mathrm{Ce}$ with an excitation energy of only $E^{*}=103(12) \mathrm{keV}$ has been discovered [84]. Left part of Figure 3 shows Schottky spectra from a recent ESR experiment where new isomeric states ${ }^{184 m 2} \mathrm{Hf},{ }^{187 m 1} \mathrm{Ta}$ and ${ }^{187 m 2}$ Ta have been discovered [85]. High resolving power of about $R>10^{6}$ achieved with Penning trap mass spectrometry allows resolving low-lying isomeric states, which has been demonstrated with a discovery of an isomeric state in ${ }^{65} \mathrm{Fe}$ at LEBIT [86]. The measured time-of-flight spectrum is illustrated in the right part of Figure 3. Mass spectrometry is a very useful tool to search for long-lived isomeric states which is complicated with the conventional $\gamma$-ray spectroscopy [87, 88].

Figure 4 illustrates the present experimental knowledge of neutron separation energies and neutron shell gaps for $N=50,82$ and 126 isotones. Plotted for comparison are the same quantities extracted from the mass models which are are frequently used in astrophysics calculations [89][92]. It is evident that more experimental data are needed to constrain the theory (see also [93, 94]). Therefore, further mass measurements of exotic nuclei are planned worldwide.

\section{Future perspectives}

Future mass measurement programs include both Penning traps and storage rings at the new generation radioactive beam facilities. It is aimed at these facilities to produce and to study very exotic nuclei, e.g., at the r-process path. For example, Penning trap facilities will profit from the highest yields of exotic nuclei at the FRIB facility in East Lansing [95] and, within the MATS project [96], at the FAIR facility in Darmstadt. At RI-Beam Factory (RIBF) in RIKEN, the RIRING project aims at the IMS mass measurements of nuclides separated with BIG-RIPS [99]. The 
planned new storage ring complex which will be constructed at the FAIR facility is illustrated in Figure 5. Here, the mass measurement program is the subject of the ILIMA project [70]. Based on the ultimate sensitivity to single stored ions, masses of nuclides with production rates as small as one per day or even per week can be measured. The nuclides which will be accessible at FAIR (assuming the smallest production rates to be 1 nucleus per day) are shown in gray in Figure 2.

\section{References}

[1] A. Bohr and B. Mottelson, Nuclear Structure (World Scient., Singapore, 1998).

[2] Yu.A. Litvinov et al., Phys. Rev. Lett. 95 (2005) 042501.

[3] R. B. Cakirli et al., Phys. Rev. Lett. 102 (2009) 082501.

[4] E. Burbidge et al. (B² FH), Rev. Mod. Phys. 29 (1957) 587.

[5] K. Blaum, Phys. Rep. 425 (2006) 1.

[6] B. Franzke, H. Geissel and G. Münzenberg, Mass Spectr. Rev. 27 (2008) 428.

[7] B. Franzke, Nucl. Instr. and Meth. B24/25 (1987) 18.

[8] J. W. Xia et al., Nucl. Instr. and Meth. A488 (2002) 11.

[9] M. Mukherjee et al., Eur. Phys. J. A35 (2008) 1.

[10] V.S. Kolhinen et al., Nucl. Instr. and Meth. A528 (2004) 776.

[11] G. Savard et al., Int. J. Mass Spectrometry 251 (2006) 252.

[12] M. Block et al., Eur. Phys. J. A25 (2005) 49.

[13] R. Ringle et al., Int. J. Mass Spectrometry 251 (2006) 300.

[14] Sz. Nagy, K. Blaum and R. Schuch, Lect. Notes Phys. 749 (2008) 1.

[15] J. Dilling et al., Int. J. Mass Spectrometry 251 (2006) 198.

[16] V.S. Kolhinen et al., Nucl. Instr. and Meth. B266 (2008) 4547.

[17] K. Blaum et al., Eur. Phys. J. A15 (2002) 245.

[18] J. Ketelaer et al., Nucl. Instr. and Meth. B594 (2008) 162.

[19] J. Ketelaer et al., Eur. Phys. J. A42 (2009) 311.

[20] Yu.A. Litvinov et al., Acta Phys. Polonica B41 (2010) 511.

[21] H. Geissel et al., Phys. Rev. Lett. 68 (1992) 3412.

[22] H. Geissel and Yu.A. Litvinov, Nucl. Instr. and Meth. B266 (2008) 4176.

[23] F. Attallah et al., Nucl. Phys. A701 (2002) 561c.

[24] T. Ohtsubo et al., Phys. Rev. Lett. 95 (2005) 052501.

[25] Yu.A. Litvinov et al., Phys. Rev. Lett. 99 (2007) 262501.

[26] Yu.A. Litvinov et al., Phys. Lett. $\mathbf{B 6 6 4}$ (2008) 162.

[27] N. Winckler et al., Phys. Lett. B679 (2009) 36.

[28] Yu.A. Litvinov and F. Bosch, Rep. Prog. Phys. 74 (2011) 016301. 
[29] N. Kalantar-Nayestanaki et al., Int. J. Mod. Phys. E18 (2009) 524.

[30] Q. Zhong et al., J. Phys. Conf. Series 202 (2010) 012011.

[31] F. Bosch, Lect. Notes Phys. 651 (2004) 137.

[32] H. Geissel, Progress in Part. and Nucl. Phys. 42 (1999) 3.

[33] F. Nolden, et al., Nucl. Instr. and Meth. A532 (2004) 329.

[34] M. Steck et al., Nucl. Instr. and Meth. A532 (2004) 357.

[35] Yu.A. Litvinov et al., Nucl. Phys. A734 (2004) 473.

[36] F. Nolden et al., Nucl. Instr. and Meth. (2010) to be published.

[37] M. Hausmann et al., Nucl. Instr. and Meth. A446 (2000) 569.

[38] T. Radon et al., Nucl. Phys. A677 (2000) 75.

[39] M. Hausmann et al., Hyperfine Interactions 132 (2001) 291.

[40] Yu.N. Novikov et al., Nucl. Phys. A697 (2002) 92.

[41] K. Blaum et al., J. Phys. Conf. Series (2010) in press.

[42] A. Wapstra, G. Audi and C. Thibault, Nucl. Phys. A729 (2003) 129.

[43] G. Sikler et al., Nucl. Phys. A763 (2005) 45.

[44] P. Delahaye et al., Phys. Rev. C74 (2006) 034331.

[45] A. Kankainen et al., Eur. Phys. J. A29 (2006) 271.

[46] A. Herlert et al., Int. J. Mass Spectrom. 251 (2006) 251.

[47] S. Rahaman et al., Eur. Phys. J. A32 (2007) 87.

[48] A. Martín et al., Eur. Phys. J. A34 (2007) 341.

[49] C. Rauth et al., Eur. Phys. J. Special Topics 150 (2007) 329.

[50] M. Dworschak et al., Phys. Rev. Lett. 100 (2008) 072501.

[51] S. Baruah et al., Phys. Rev. Lett. 101 (2008) 262501.

[52] J. Hakala et al., Phys. Rev. Lett. 101 (2008) 052501.

[53] C. Weber et al., Nucl. Phys. $\mathbf{8 8 0 3}$ (2008) 1.

[54] C. Weber et al., Phys. Rev. C78 (2008) 054310.

[55] V.-V. Elomaa et al., Eur. Phys. J. A40 (2009) 1.

[56] M. Breitenfeldt et al., Phys. Rev. C80 (2009) 035805.

[57] D. Neidherr et al., Phys. Rev. C80 (2009) 044323.

[58] M. Kowalska et al., Eur. Phys. J. A42 (2009) 351.

[59] V.-V. Elomaa et al., Phys. Rev. Lett. 102 (2009) 252501.

[60] U. Hager et al., Phys. Rev. Lett. 96 (2006) 042504.

[61] A. Kankainen et al., Phys. Rev. Lett. 101 (2008) 142503.

[62] D. Rodríguez et al., Phys. Rev. Lett. 93 (2004) 161104. 
[63] M. Block et al., Nature 463 (2010) 785.

[64] Yu.A. Litvinov, Int. J. Modern Phys. E18 (2009) 323.

[65] J. Stadlmann et al., Phys. Lett. $\mathbf{B 5 8 6}$ (2004) 27.

[66] B. Sun et al., Int. J. Modern Phys. E18 (2009) 346.

[67] B. Sun et al., Nucl. Phys. A812 (2008) 1.

[68] L. Chen, Ph.D. Thesis, University of Giessen (2008).

[69] Yu.A. Litvinov et al., Nucl. Phys. A756 (2005) 3.

[70] P.M. Walker et al., Technical proposal for the ILIMA project, Report, GSI (2005).

[71] Yu.A. Litvinov et al., Hyperfine Interactions 173 (2006) 55.

[72] H. Geissel et al., Hyperfine Interactions 173 (2006) 49.

[73] X. Tu et al., Chinese Phys C33 (2009) 516.

[74] H. Xu et al., Chinese Science Bulletin 54 (2009) 4749.

[75] S. Rahaman et al., Phys. Rev. Lett. 103 (2009) 042501.

[76] T. Eronen et al., Phys. Rev. Lett. 103 (2009) 252501.

[77] F. Bosch et al., Int. J. Mass Spectrometry 251 (2006) 212.

[78] L. Chen et al., Phys. Lett. $\mathbf{B 6 9 1}$ (2010) 234.

[79] D. Neidherr et al., Phys. Rev. Lett. 102 (2009) 112501.

[80] Yu.A. Litvinov et al., Hyperfine Interactions 132 (2001) 283.

[81] H. Geissel et al., Nucl. Phys. $\mathbf{A 6 8 5}$ (2001) 115c.

[82] L. Chen et al., Phys. Rev. Lett. 102 (2009) 122503.

[83] H. Geissel and Yu.A. Litvinov, J. Phys. G31 (2005) S1779.

[84] B. Sun et al., Eur. Phys. J. A31 (2007) 393.

[85] M.W. Reed et al., Phys. Rev. Lett. 105 (2010) 172501.

[86] M. Block et al., Phys. Rev. Lett. 100 (2008) 132501.

[87] P.M. Walker and G.D. Dracoulis, Hyperfine Interactions 135 (2001) 83.

[88] P.M. Walker and G.D. Dracoulis, Nature 399 (1999) 35.

[89] P. Möller, J.R. Nix, W.D. Myers and W.J. Swiatecki, At. Data Nucl. Data Tables 59 (1995) 185.

[90] J. Duflo and A.P. Zuker, Phys. Rev. C66 (2002) 051304(R).

[91] S. Goriely, M. Samyn and J.M. Pearson, Phys. Rev. C75 (2007) 064312.

[92] S. Goriely, N. Chamel and J.M. Pearson, Phys. Rev. Lett. 102 (2009) 152503.

[93] H. Geissel et al., Nucl. Phys. $\mathbf{A 7 4 6}$ (2004) 150c.

[94] B. Sun et al., Phys. Rev. C78 (2008) 025806.

[95] The Facility for Rare-Isotope Beams (FRIB), http://www.frib.msu.edu/.

[96] D. Rodríguez et al., Eur. Phys. J. Special Topics 183 (2010) 1.

[97] A. Dolinskii, et al., Nucl. Instr. and Meth. A 574, 207 (2007).

[98] C. Scheidenberger et al., Hyperfine Interactions 173 (2006) 61.

[99] Y. Yamaguchi et al., Nucl. Instr. and Meth. B266 (2008) 4575. 